\title{
On The Circulant K-Fibonacci Matrices
}

\author{
Sergio Falcon ${ }^{1}$

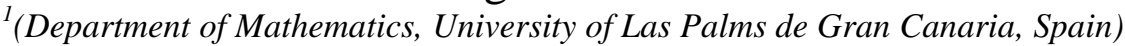

\begin{abstract}
We started looking for a formula to simplify the calculation of the difference of two k-Fibonacci numbers depending on the kind of subscripts. Then we study the value of the determinant of circulant matrices whose entries are $k$-Fibonacci numbers. We continue calculating their eigenvalues and finish with the calculation of the eigenvalues of the matrix obtained multiplying the $k-$ Fibonacci
\end{abstract}

Keywords: $k$-Fibonacci and k-Lucas numbers, Eigenvalues, Circulant matrix.

\section{Introduction}

The classical Fibonacci sequence $\{0,1,1,2,3,5,8 \ldots\}$ had been extended in many ways [1,2]. One on which they are working more intensely in recent years is due to Falcon and Plaza $[3,4]$ which we remember. For a given integer number k, we define the k-Fibonacci sequence $F_{k}=\left\{F_{k, n}\right\}_{n \in N}$ by the recurrence relation $F_{k, n+1}=k F_{k, n}+F_{k, n-1}$ for $\mathrm{n} \geq 1$ with initial conditions $F_{k, 0}=0, F_{k, 1}=1$.

According to this definition, the general expression of the first terms of the k-Fibonacci sequence are $F_{k}=\left\{0,1, k, k^{2}+1, k^{3}+2 k, k^{4}+3 k^{2}+1 \ldots\right\}$. In particular, for $\mathrm{k}=1$ the classical Fibonacci sequence $F_{1}=F=\{0,1,1,2,3,5,8 \ldots\}$ is obtained while for $\mathrm{k}=2$ we get the Pell sequence $F_{2}=\{0,1,2,5,12,29,70,169 \ldots\}$.

Characteristic equation of this sequence is $r^{2}=k \cdot r+1$ whose solutions are $\sigma_{1}=\frac{k+\sqrt{k^{2}+4}}{2}$ and $\sigma_{2}=\frac{k-\sqrt{k^{2}+4}}{2}$. It is easy to prove these solutions verify

$\sigma_{1} \cdot \sigma_{2}=-1, \sigma_{1}+\sigma_{2}=k, \sigma_{1}-\sigma_{2}=\sqrt{k^{2}+4}, \sigma^{2}=k \sigma+1, \sigma_{1}>0, \sigma_{2}<0$.

In particular, the Binet Identity for the k-Fibonacci numbers is $F_{k, n}=\frac{\sigma_{1}^{n}-\sigma_{2}^{n}}{\sigma_{1}-\sigma_{2}}$.

Moreover, we define the k-Fibonacci numbers with negative subscript as $F_{k,-n}=(-1)^{n+1} F_{k, n}$.

Similarly, we define the k-Lucas numbers as $L_{k, n+1}=k \cdot L_{k, n}+L_{k, n-1}$ with initial conditions $L_{k, 0}=2, L_{k, 1}=k$. [5]. The Binet Identity for the k-Lucas numbers takes the form $L_{k, n}=\sigma_{1}^{n}+\sigma_{2}^{n}$ and consequently $L_{k, n}=F_{k, n-1}+F_{k, n+1}$. Moreover, $L_{k,-n}=(-1)^{n} L_{k, n}$.

With these instructions, it is relatively easy to prove

$$
\sum_{j=0}^{n} F_{k, r+j}^{2}=\frac{1}{k\left(k^{2}+4\right)}\left(L_{k, 2 r+2 n+1}-L_{k, 2 r-1}+(-1)^{r}\left((-1)^{n+1}-1\right)\right)
$$

Now, as we will later need this formula, we will simplify $F_{k, p+m}-F_{k, p-m}$ according to $m$ whether it is even or odd. From the Binet Identity and taking into account $\sigma_{1} \cdot \sigma_{2}=-1$,

$$
F_{k, p+m}-F_{k, p-m}=\frac{\sigma_{1}^{p+m}-\sigma_{2}^{p+m}}{\sigma_{1}-\sigma_{2}}-\frac{\sigma_{1}^{p-m}-\sigma_{2}^{p-m}}{\sigma_{1}-\sigma_{2}}=\frac{1}{\sigma_{1}-\sigma_{2}}\left[\sigma_{1}^{p}\left(\sigma_{1}^{m}-\frac{1}{\sigma_{1}^{m}}\right)-\sigma_{2}^{p}\left(\sigma_{2}^{m}-\frac{1}{\sigma_{2}^{m}}\right)\right]
$$

- m even: $F_{k, p+m}-F_{k, p-m}=\frac{1}{\sigma_{1}-\sigma_{2}}\left[\sigma_{1}^{p}\left(\sigma_{1}^{m}-\sigma_{2}^{m}\right)-\sigma_{2}^{p}\left(\sigma_{2}^{m}-\sigma_{1}^{m}\right)\right]=\frac{\sigma_{1}^{m}-\sigma_{2}^{m}}{\sigma_{1}-\sigma_{2}}\left(\sigma_{1}^{p}+\sigma_{2}^{p}\right)=F_{k, m} L_{k, p}$

- $\quad$ odd: $F_{k, p+m}-F_{k, p-m}=\frac{1}{\sigma_{1}-\sigma_{2}}\left[\sigma_{1}^{p}\left(\sigma_{1}^{m}+\sigma_{2}^{m}\right)-\sigma_{2}^{p}\left(\sigma_{2}^{m}+\sigma_{1}^{m}\right)\right]=\frac{\sigma_{1}^{p}-\sigma_{2}^{p}}{\sigma_{1}-\sigma_{2}}\left(\sigma_{1}^{m}+\sigma_{2}^{m}\right)=F_{k, p} L_{k, m}$ 
In short:

$$
F_{k, p+m}-F_{k, p-m}=\left\{\begin{array}{l}
F_{k, m} L_{k, p}, \text { if } m \text { is even } \\
F_{k, p} L_{k, m}, \text { if } m \text { is odd }
\end{array}\right.
$$

\subsection{Matrix norms}

The following matrix norms are defined in $[6,7]$.

Let $A=\left(a_{i j}\right)$ be an $\mathrm{m} \times \mathrm{n}$ matrix.

- The Frobenius or Euclidean norm of A is defined as $\|A\|_{E}=\left(\sum_{i=1}^{m} \sum_{j=1}^{n}\left|a_{i j}\right|^{2}\right)^{1 / 2}$

- The column norm of $\mathrm{A}$ is defined as $\|A\|_{1}=\max _{1 \leq j \leq n} \sum_{i=1}^{m}\left|a_{i j}\right|$, which is simply the maximum absolute column sum of the matrix.

- The row norm of $\mathrm{A}$ is $\|A\|_{\infty}=\max _{1 \leq j \leq m} \sum_{i=1}^{n}\left|a_{i j}\right|$, which is simply the maximum absolute row sum of the matrix.

- The spectral norm of a matrix A is the largest singular value of A i.e. the square root of the largest eigenvalue of the positive-semidefinite matrix $A^{*} A$ where $A^{*}$ denotes the conjugate transpose of $\mathrm{A}$; that is $\|A\|_{2}=\sqrt{\lambda_{\max }\left(A^{*} A\right)}=\sigma_{\max }(A)$

\subsection{Circulant matrix}

Given the n numbers $\left\{a_{0}, a_{1}, a_{2} \ldots a_{n-1}\right\}$, the matrix $C_{n}=\left(\begin{array}{ccccc}a_{0} & a_{1} & a_{2} & \cdots & a_{n-1} \\ a_{n-1} & a_{0} & a_{1} & \cdots & a_{n-2} \\ a_{n-2} & a_{n-1} & a_{0} & \cdots & a_{n-3} \\ \vdots & \vdots & \vdots & \vdots & \vdots \\ a_{1} & a_{2} & a_{3} & \cdots & a_{0}\end{array}\right)$ is called a circulant matrix

$[8,9,10]$, because the entry $\{i, j\}$ is equal to the entry $\{i+l, j+l\}$ for $l=1,2, \ldots$ If $C_{n}$ is a circulant matrix, its transpose matrix $\left(C_{n}\right)^{T}$ is also circulant.

It is known the determinant of the circulant matrix $C_{n}$ is [8]

$$
\operatorname{det}\left(C_{n}\right)=\left|C_{n}\right|=\prod_{l=0}^{n-1}\left(\sum_{j=0}^{n-1} a_{j} w_{l}^{j}\right)
$$

where $w_{l}=e^{\frac{2 \pi l}{n} i}$ are the $\mathrm{n}^{\text {th }}$ roots of unity.

We will use the notation $C=\operatorname{CIRC}\left(a_{0}, a_{1}, a_{2} \ldots a_{n-1}\right)$ for the $\mathrm{n} \times \mathrm{n}$ circulant matrix whose top row is $c=\left\{a_{0}, a_{1}, a_{2} \ldots a_{n-1}\right\}$.

And later we will need the following properties:

a) The map $\lambda: \operatorname{CIRC}_{n}(\square) \rightarrow \square^{n}$ is the eigenvalue map on real $\mathrm{n} \times \mathrm{n}$ circulant matrices to complex $\mathrm{n}$-vectors.

Thus, if $C \in C I R C(\square)$, then $\lambda(C)$ is the set of $\mathrm{n}$ eigenvalues of the matrix $\mathrm{C}$.

b) $\lambda_{i}\left(\operatorname{CIRC}\left(a_{0}, a_{1} \ldots a_{n-1}\right)=\sum_{j=0}^{n-1} a_{j} w_{j}^{l} \quad\right.$ ([11], Theorem 1.6(ii)).

c) $\lambda$ is an algebra isomorphism ([11], Corollary 1.8.1).

For the norms of circulant matrices, see [12, 13, $14-18]$.

\subsection{Proposition}

If $a, b \in \square, b \neq 0$ and $a+i b$ is an eigenvalue of a real circulant matrix $A$, then $a^{2}+b^{2}$ is an eigenvalue of the product matrix $A \cdot A^{T}$ with multiplicity $\geq 2$, where $A^{T}$ is the transpose matrix of $A$.

Proof.

Suppose $A=\operatorname{CIRC}\left(a_{0}, a_{1} \ldots a_{n-1}\right)$. Then $A^{T}=\operatorname{CIRC}\left(a_{0}, a_{n-1}, a_{n-2} \ldots a_{1}\right)$. 
We are given that $a+i b=\lambda_{i}(A)$ for some i, $0 \leq i \leq n$, with $b \neq 0$. Therefore, $a-i b=\lambda_{n-i}(A)$ is also an eigenvalue for the above Property (c). (if the subscript i is $\mathrm{n}-\mathrm{i}$, then $\mathrm{b}=0$ contrary to what is given).

Again for the Property (c), $\lambda_{i}\left(A^{T}\right)=a-i b$ and $\lambda_{n-i}\left(A^{T}\right)=a+i b$.

Hence $\lambda_{i}\left(A A^{T}\right)=\lambda_{n-i}\left(A A^{T}\right)=a^{2}+b^{2}$ and its multiplicity is $\geq 2$.

The proof still works in case $\mathrm{b}=0$ provided $\mathrm{n}$ is odd and a $a \neq \lambda_{0}(A)=\sum_{j=0}^{n-1} a_{j}$, otherwise if, for example, $\mathrm{b}=0$ and $\mathrm{n}$ is even, the eigenvalue $a^{2}$ can be non-degenerate in AAT . But, in this case, the multiplicity is 1 because the eigenvalue is $\lambda_{i}=a \pm 0 i$ with multiplicity 1 .

\section{A Circulant K-Fibonacci Matrix}

According to previous definition, for $\mathrm{r} \geq 0,\left(C F_{k}\right)_{n, r}=\left(\begin{array}{ccccc}F_{k, r} & F_{k, r+1} & F_{k, r+2} & \cdots & F_{k, r+n-1} \\ F_{k, r+n-1} & F_{k, r} & F_{k, r+1} & \cdots & F_{k, r+n-2} \\ F_{k, r+n-2} & F_{k, r+n-1} & F_{k, r} & \cdots & F_{k, r+n-3} \\ \vdots & \vdots & \vdots & \vdots & \vdots \\ F_{k, r+1} & F_{k, r+2} & F_{k, r+3} & \cdots & F_{k, r}\end{array}\right)$ is called circulant k-Fibonacci matrix.

Next we try to simplify the expression of the determinant of this matrix. It is obvious that $n>1$ or $r>0$, it is $\left|\left(C F_{k}\right)_{n, r}\right| \neq 0$

\subsection{Theorem (Determinant of the $\mathbf{k}$-Fibonacci circulant matrix)}

The value of the circulant $k-$ Fibonacci determinant is

$$
\left|\left(C F_{k}\right)_{n, r}\right|=\frac{\left(F_{k, r+n-1}-F_{k, r-1}\right)^{n}-\left(F_{k, r}-F_{k, r+n}\right)^{n}}{L_{k, n}-1-(-1)^{n}}
$$

Proof.

According to Formula (1.3), $\left|\left(C F_{k}\right)_{n, r}\right|=\prod_{l=0}^{n-1}\left(\sum_{j=0}^{n-1} F_{k, r+j} w_{l}^{j}\right)$. Then

$$
\begin{aligned}
\left|\left(C F_{k}\right)_{n, r}\right| & =\prod_{l=0}^{n-1}\left(\sum_{j=0}^{n-1} \frac{\sigma_{1}^{r+j}-\sigma_{2}^{r+j}}{\sigma_{1}-\sigma_{2}} w_{l}^{j}\right)=\prod_{l=0}^{n-1}\left(\sum_{j=0}^{n-1} \frac{1}{\sigma_{1}-\sigma_{2}}\left(\sigma_{1}^{r}\left(\sigma_{1} w_{l}\right)^{j}-\sigma_{2}^{r}\left(\sigma_{2} w_{l}\right)^{j}\right) w_{l}^{j}\right) \\
& =\prod_{l=0}^{n-1} \frac{1}{\sigma_{1}-\sigma_{2}}\left(\sigma_{1}^{r} \frac{\left(\sigma_{1} w_{l}\right)^{n}-1}{\sigma_{1} w_{l}-1}-\sigma_{2}^{r} \frac{\left(\sigma_{2} w_{l}\right)^{n}-1}{\sigma_{2} w_{l}-1}\right) \\
& =\prod_{l=0}^{n-1} \frac{1}{\sigma_{1}-\sigma_{2}}\left(\frac{\sigma_{1}^{r}\left(\sigma_{1}^{n} w_{l}^{n}-1\right)\left(\sigma_{2} w_{l}-1\right)-\sigma_{2}^{r}\left(\sigma_{2}^{n} w_{l}^{n}-1\right)\left(\sigma_{1} w_{l}-1\right)}{\left(\sigma_{1} w_{l}-1\right)\left(\sigma_{2} w_{l}-1\right)}\right) \\
& =\prod_{l=0}^{n-1} \frac{\left(\sigma_{1}^{r+n} w_{l}^{n}-\sigma_{1}^{r}\right)\left(\sigma_{2} w_{l}-1\right)-\left(\sigma_{2}^{r+n} w_{l}^{n}-\sigma_{2}^{r}\right)\left(\sigma_{2} w_{l}-1\right)}{\left(\sigma_{1}-\sigma_{2}\right)\left(\sigma_{1} w_{l}-1\right)\left(\sigma_{2} w_{l}-1\right)} \\
& =\coprod_{l=0}^{n-1} \frac{\sigma_{1}^{r+n} \sigma_{2} w_{j}^{n+1}-\sigma_{1}^{r+n} w_{l}^{n}-\sigma_{1}^{r} \sigma_{2} w_{l}+\sigma_{1}^{r}-\sigma_{2}^{r+n} \sigma_{1} w_{j}^{n+1}-\sigma_{2}^{r+n} w_{l}^{n}-\sigma_{2}^{r} \sigma_{1} w_{l}+\sigma_{2}^{r}}{\left(\sigma_{1}-\sigma_{2}\right)\left(\sigma_{1} w_{l}-1\right)\left(\sigma_{2} w_{l}-1\right)} \\
& =\prod_{l=0}^{n-1} \frac{\left(\sigma_{1}^{r}-\sigma_{2}^{r}\right)-\left(\sigma_{1}^{r+n}-\sigma_{2}^{r+n}\right)+\left(\left(\sigma_{1}^{r-1}-\sigma_{2}^{r-1}\right)-\left(\sigma_{1}^{r+n-1}-\sigma_{2}^{r+n-1}\right)\right) w_{l}}{\left(\sigma_{1}-\sigma_{2}\right)\left(\sigma_{1} w_{l}-1\right)\left(\sigma_{2} w_{l}-1\right)} \\
& =\prod_{l=0}^{n-1} \frac{F_{k, r}-F_{k, r+n}+\left(F_{k, r-1}-F_{k, r+n-1}\right) w_{l}}{\left(\sigma_{1} w_{l}-1\right)\left(\sigma_{2} w_{l}-1\right)}
\end{aligned}
$$

because $w_{l}^{n}=1$ and $\sigma_{1} \sigma_{2}=-1$. 
On the other hand, $\prod_{l=0}^{n-1}\left(a-b w_{l}\right)=b^{n} \prod_{l=0}^{n-1}\left(\frac{a}{b}-w_{l}\right)=b^{n}\left(\left(\frac{a}{b}\right)^{n}-1\right)=a^{n}-b^{n}$. Therefore

- $\quad \prod_{l=0}^{n-1}\left(F_{k, r}-F_{k, r+n}-\left(F_{k, r+n-1}-F_{k, r-1}\right) w_{l}\right)=\left(F_{k, r}-F_{k, r+n}\right)^{n}-\left(F_{k, r+n-1}-F_{k, r-1}\right)^{n}$

- $\prod_{l=0}^{n-1}\left(\sigma_{1} w_{l}-1\right)\left(\sigma_{2} w_{l}-1\right)=\prod_{l=0}^{n-1}\left(\sigma_{1} w_{l}-1\right) \prod_{l=0}^{n-1}\left(\sigma_{2} w_{l}-1\right)=\left(\sigma_{1}^{n}-1\right)\left(\sigma_{2}^{n}-1\right)=1-\left(\sigma_{1}^{n}+\sigma_{2}^{n}\right)+(-1)^{n}$

$$
=1-L_{k, n}+(-1)^{n}
$$

Consequently, this establishes the equation (1.4).

From this equation, $\left|\left(C F_{k}\right)_{n, r}\right|$ is positive or negative according $\mathrm{n}$ is odd or even, respectively.

This formula can be simplified if $\mathrm{n}$ is even. Comparing the formulas (2) and (4) it is $m=\frac{n}{2}$. Then,

- $\mathrm{m}$ is even if $\mathrm{n} \equiv 0$ (mod 4) and then $\left|\left(C F_{k}\right)_{n, r}\right|=\frac{\left(F_{k, \frac{n}{2}} L_{k, r+1+\frac{n}{2}}\right)^{n}-\left(F_{k,-\frac{n}{2}} L_{k, r+\frac{n}{2}}\right)^{n}}{L_{k, n}-2}=\frac{F_{k, \frac{n}{2}}^{n}\left(L_{k, r-1+\frac{n}{2}}^{n}-L_{k, r+\frac{n}{2}}^{n}\right)}{L_{k, n}-2}$

- $\quad m$ is odd if $n \equiv 2(\bmod 4)$ and then

$$
\left|\left(C F_{k}\right)_{n, r}\right|=\frac{\left(L_{k, \frac{n}{2}} F_{k, r-1+\frac{n}{2}}\right)^{n}-\left(L_{k,-\frac{n}{2}} F_{k, r+\frac{n}{2}}\right)^{n}}{L_{k, n}-2}=\frac{L_{k, \frac{n}{2}}^{n}\left(F_{k, r-1+\frac{n}{2}}^{n}-F_{k, r+\frac{n}{2}}^{n}\right)}{L_{k, n}-2}
$$

\subsection{Matrix norms of the $k$-Fibonacci circulant matrix}

Taking into account the definition of the Euclidean matrix norm, and as all the row vectors have the same entries, the Euclidean norm of the k-Fibonacci circulant matrix is $\left\|\left(C F_{k}\right)_{n, r}\right\|_{E}=n \sum_{j=0}^{n-1} F_{k, r+j}^{2}$. And applying the formula (1), it is $\left\|\left(C F_{k}\right)_{n, r}\right\|_{E}^{2}=\frac{n}{k\left(k^{2}+4\right)}\left(L_{k, 2 r+2 n-1}-L_{k, 2 r-1}+(-1)^{r+n}-(-1)^{r}\right)$.

Logically, the Euclidean norm of the k-Fibonacci circulant matrix is $n$ times its row or its column norm.

\subsection{Eigenvalues and eigenvectors}

The eigenvalues of $\left(C F_{k}\right)_{n, r}$ are given by $\lambda_{j}=\sum_{l=0}^{n-1} F_{k, r+l} w_{j}^{l}[11,10]$, where $w_{j}=\exp \left(\frac{2 \pi i}{n} j\right)$ are the $\mathrm{n}-$ th roots of the unity and $i$ is the imaginary unit.

The corresponding normalized eigenvectors are given by $\vec{e}=\frac{1}{\sqrt{n}}\left(1, w_{j}, w_{j}^{2} \ldots w_{j}^{n-1}\right)^{T}, j=0,1,2 \ldots n-1$.

Taking into account if $p \neq q \rightarrow F_{k, p} \neq F_{k, q}$, the eigenvalues of $\left(C F_{k}\right)_{n, r}$ verify the following properties:

(1) All the eigenvalues are simple.

(2) If $\mathrm{n}$ is odd, only one eigenvalue is real: $\lambda_{0}=\sum_{l=0}^{n-1} F_{k, r+j}$.

(3) If $\mathrm{n}$ is even, $\mathrm{n}=2 \mathrm{p}$, the matrix $\left(C F_{k}\right)_{n, r}$ get only two real eigenvalues: $\lambda_{0}$ and $\lambda_{p}=\sum_{l=0}^{n-1}(-1)^{j} F_{k, r+j}$

(4) Half the other eigenvalues of $\left(C F_{k}\right)_{n, r}$ gets complex and the other half are their conjugates.

For instance, if $\mathrm{n}=3$, the eigenvalues of $\left(C F_{k}\right)_{3, r}$ are:

1) $w_{0}=1 \rightarrow \lambda_{0}=F_{k, r}+F_{k, r+1}+F_{k, r+2}$

2) $w_{1}=-\frac{1}{2}+i \frac{\sqrt{3}}{2} \rightarrow \lambda_{1}=F_{k, r}+F_{k, r+1}\left(-\frac{1}{2}+i \frac{\sqrt{3}}{2}\right)+F_{k, r+2}\left(-\frac{1}{2}-i \frac{\sqrt{3}}{2}\right)$ 
3) $w_{2}=-\frac{1}{2}-i \frac{\sqrt{3}}{2} \rightarrow \lambda_{1}=F_{k, r}+F_{k, r+1}\left(-\frac{1}{2}-i \frac{\sqrt{3}}{2}\right)+F_{k, r+2}\left(-\frac{1}{2}+i \frac{\sqrt{3}}{2}\right)$

Evidently, $\lambda_{2}=\bar{\lambda}_{1}$

\section{On The Matrix Product $\left(C F_{k}\right)_{n, r} \cdot\left(\left(C F_{k}\right)_{n, r}\right)^{T}$}

Let us consider the matrix $M_{n, r}=\left(C F_{k}\right)_{n, r} \cdot\left(\left(C F_{k}\right)_{n, r}\right)^{T}$, where $\left(\left(C F_{k}\right)_{n, r}\right)^{T}((\mathrm{CFk}) \mathrm{n}, \mathrm{r}) \mathrm{T}$ is the transpose matrix of $\left(C F_{k}\right)_{n, r}$. Evidently, $M_{n, r}$ is double symmetric, that is $a_{i, j}=a_{j, i}$ and $a_{i, j}=a_{i+l, j+l}$. Consequently, all its eigenvalues are real. Finally, $M_{n, r}$ is also circulant.

If $\vec{a}_{1}=\left\{a_{1, c}\right\}, c=1,2 \ldots n-1$ is the first row vector of this matrix, then

$c=1: a_{1,1}=\sum_{j=0}^{n-1} F_{k, r+j}^{2}$

$c>1 ; \quad a_{1, c}=\sum_{j=0}^{c-2} F_{k, r+j} F_{k, r+n+j-(c-1)}+\sum_{j=c-1}^{n-1} F_{k, r+j} F_{k, r+j-(c-1)}$

Taking into account Proposition 1, we can deduce the following theorem.

\subsection{Theorem}

If $\lambda$ is an eigenvalue of the circulant matrix $\left(C F_{k}\right)_{n, r}$, the square of its norm, $|\lambda|^{2}$, is an eigenvalue of $M_{n, r}=\left(C F_{k}\right)_{n, r} \cdot\left(\left(C F_{k}\right)_{n, r}\right)^{T}$.

\subsection{Corollary}

If $\lambda=a+i b, b \neq 0$ is a complex eigenvalue of $\left(C F_{k}\right)_{n, r}$ then $|\lambda|^{2}=a^{2}+b^{2}$ is a double eigenvalue of $M_{n, r}=\left(C F_{k}\right)_{n, r} \cdot\left(\left(C F_{k}\right)_{n, r}\right)^{T}$.

If $\lambda=\mathrm{a}$ is a real eigenvalue of $\left(C F_{k}\right)_{n, r}$, then $\lambda^{2}$ is a simple eigenvalue of $M_{n, r}=\left(C F_{k}\right)_{n, r} \cdot\left(\left(C F_{k}\right)_{n, r}\right)^{T}$.

\section{Acknowledgements}

I thank AlunWyn-jones the suggestions made for the publication of this article as well as the proof of Proposition 1. This research did not receive any specific grant from funding agencies in the public, commercial, or not-for-profit sectors.

\section{References}

[1] V.E. Hoggat, Fibonacci and Lucas numbers, Palo Alto, CA: Houghton-Mifflin; 1969.

[2] A.F. Horadam, A generalized Fibonacci sequence, Math. Mag., Vol. 68, 1961, 455-459.

[3] Sergio Falcon, A. Plaza, On the Fibonacci k-numbers, Chaos, Solit. \& Fract., Vol. 32(5), 2007, $1615-24$

[4] Sergio Falcon, A. Plaza, The k-Fibonacci sequence and the Pascal 2-triangle, Chaos, Solit. \& Fract., Vol. 33(1), 2007, 38-49.

[5] Sergio Falcon, On the k-Lucas numbers, Int. J. Contemp. Math. Sciences, Vol. 6(21), 2011, 1039-1050.

[6] Simon Foucart, http://www.math.drexel.edu/foucart/TeachingFiles/F12/M504Lect6.pdf

[7] http://en.wikipedia.org/wiki/Matrix norm

[8] Irwin Kra and Santiago R. Simanca, On circulant matrices, http://www.math.columbia.edu/ums/pdf/cir-not5.pdf

[9] D.A. Lind, A Fibonacci circulant, Fibonacci Quarterly, Vol. 8(5), 1970, 449-455.

[10] http://www.circulants.org/circ/circall.pdf

[11] Alun Wyn-jones, Circulants, www.circulants.org/circ/circall.pdf

[12] D. Bozkurt. On the spectral norms of the matrices connected to integer number sequences. Applied Mathematics and Computation, 219, (2013), 6576-6579.

[13] Philip Davis. Circulant Matrices. John Wiley \& Sons Inc, New York, 1979.

[14] E. Gokcen Alptekin, T. Mansour, and N. Tuglu, Norms of Circulant and Semicirculant matrices and Horadams sequence. Ars combinatoria, 85, (2007), 353-359.

[15] S. Shen, J. Cen. On the Spectral Norms of r-Circulant Matrices with the k-Fibonacci and k-Lucas Numbers. Int. J. Contemp. Math. Sciences, 5(12), 2010, 569-578.

[16] Y. Yazlik, N. Taskara. On the inverse of circulant matrix via generalized k-Horadam numbers. Applied Mathematics and Computation, 223, (2013), 191-196.

[17] J. Zhou. The Identical Estimates of Spectral Norms for Circulant Matrices with Binomial Coefficients Combined with Fibonacci Numbers and Lucas Numbers Entries. Journal of Function Spaces, Volume 2014, Article ID 672398, 5 pages.

[18] J. Zhou. The spectral norms of g-circulant matrices with classical Fibonacci and Lucas numbers entries. Applied Mathematics and Computation, 233, (2014), 582-587. 